\title{
The generality of mental addition models: Simple and complex addition in a decision-production task
}

\author{
LUC TIMMERS and WILLEM CLAEYS \\ University of Leuven, Leuven, Belgium
}

\begin{abstract}
Recently, Widaman, Geary, Cormier, and Little (in press) offered a general componential model for simple and complex addition. In the present study, the generalizability of this model to very complex addition problems and to production task performance was tested. We investigated addition-production performance using a new research paradigm, the decision-production task. The paradigm was used to assess the reaction times of 16 adults to 100 single-digit addition problems (Experiment 1) and to very complex three-digit addition problems (Experiment 2). Generally, the results give evidence for the basic assumptions of the Widaman et al. model. Some of the results, however, demonstrate that short-term memory load factors and strategy choices also have important effects on complex addition-production performance. Furthermore, the results of both experiments indicate that, in production tasks, the nature of the elementary search/compute process can be characterized as a memory-retrieval process and that the decision-production paradigm is a valuable alternative to the classical verbal production paradigm.
\end{abstract}

Recently, Widaman, Geary, Cormier, and Little (1989) presented a general componential model for mental addition. The model was validated for simple and moderately simple addition problems presented in a verification task. In the present study, we demonstrate the generalizability of this componential addition model (Widaman et al., 1989) to more complex addition problems and to productiontask performance.

Besides the testing of the general componential model, we also wanted to assess the generalizability of some previous findings in the mental addition area. (e.g., Ashcraft \& Stazyk, 1981; Geary, Widaman, \& Little, 1986; Widaman et al., 1989) to production-task performance. Particularly, we were interested in clarifying the nature of one of the basic component processes in mental addition: the search/compute process. This process leads to the generation of the so-called "whole number" facts $0+0$ through $9+9$; it is responsible for the well-known problem-size effect in mental addition. A lot of evidence-mainly gathered in verification-task studies-gives support for the memory-retrieval nature of the search/ compute process, at least in adult performance. Baroody $(1983,1984,1985)$, however, questioned the generalizability of this finding to production-task performance.

A further objective of the present study involved the evaluation of a new research paradigm, the decisionproduction paradigm. This paradigm differs from the clas-

We would like to thank David Geary and an anonymous reviewer for their comments on an earlier version of this article. Correspondence regarding this article may be addressed to Luc Timmers, Department of Psychology, University of Leuven, Tiensestraat 102, B-3000 Leuven, Belgium. sical verification paradigm, as well as from the production paradigm. In essence, however, the decision-production task is a production task: The subject must produce the answer to an addition problem, but, before doing this, he/she must make a decision about an aspect of the generated answer.

\section{A COMPONENTIAL MODEL FOR MENTAL ADDITION}

In the Widaman et al. (1989) model, which is an elaboration of Ashcraft's model (Ashcraft \& Battaglia, 1978), five different component processes, or stages, are distinguished: encoding, search/compute, carry, decision, and response. The processes are assumed to operate serially and independently when solving an addition task. The first component involves the encoding of the digits of the addition problem, and it results in a representation of the problem in working memory. In the search/compute stage, a sum for the basic addition problems (the problems $0+0$ through $9+9$ ) is obtained by either a counting or a memory search process. The decision stage only appears in verification tasks and involves a comparison between the computed or retrieved sum and the given sum; it results in a true/false decision. In the response stage, the overt response is prepared and executed.

The carry component, which was added to the model so that it could account for complex, as well as simple, addition problems (Geary \& Widaman, 1987; Geary, Widaman, \& Little, 1986), can only appear in complex or multidigit problems and assumes that complex problems presented in a vertical format are processed columnwise. To incorporate the columnwise-processing assumption 
into the model, a recycling loop is hypothesized. This recycling loop involves the recycling of the encoding and search/compute processes until all columns have been processed. The carry operation is required if the preceding column result is greater than nine. Widaman et al. (1989) also incorporated into their model an assumption of self-terminating processing for complex multicolumn problems: It was assumed that processing is terminated and the response "false" is executed when the first columnwise error is encountered.

Using the verification paradigm, Widaman et al. (1989) demonstrated the internal validity of the proposed componential model for simple and moderately complex addition problems. Their results also supported the hypothesis that the search/compute stage can be characterized as a memory-retrieval process: Reaction times (RTs) to the addition problems were predicted best when the search/ compute process was represented by the "product of the addends" (Prod) structural variable. The Prod model is generally interpreted in terms of a memory network model in which it is assumed that the calculation of a sum involves a search and retrieval of the sum in a long-term memory network of addition facts.

Widaman et al. (1989) conceptualized the memory network as a table-like, square, symmetric matrix, with the row and column axes representing the entry nodes and the values 0 through 9 representing the two addends of an addition problem. The correct sum of any two numbers is stored at the point of intersection of the two entry nodal values. Furthermore, Widaman et al. assumed that, when an addition problem is presented, the origin node is activated and, by means of a mechanism of spreading activation (Anderson, 1983; Collins \& Loftus, 1975), associatively connected nodes are activated until the intersection node is activated. The rate of activation of the memory network is a constant function of the area of the network activated. The product structural variable represents the total area of the matrix activated and is therefore linearly related to search time or RT required to arrive at the correct answer.

Alternative conceptualizations of the memory network model have been presented in the literature, starting from the high predictivity of variables other than the Prod variable (e.g., "the square of the sum" [Sum²]). Irrespective of these slightly different conceptualizations of the memory-retrieval process, the results of a large majority of studies on mental addition-especially on adult mental addition-indicate that, in the search/compute stage, a memory-retrieval process is used, rather than counting or analog processes (which are associated with structural variables, such as the minimum or maximum addend). For example, a series of studies demonstrate arithmetic priming and interference effects that can be explained only by a memory model (e.g., Ashcraft \& Battaglia, 1978; Campbell, 1987a, 1987b; LeFevre, Bisanz, \& Mrkonjic, 1988; Stazyk, Ashcraft, \& Hamann, 1982), and studies interested in predicting RTs (see, e.g., Svenson, 1985) show that adult RT is predicted better by a memoryretrieval process (represented by a Prod or Sum² model) than by counting or analog processes. An exhaustive description and discussion of the counting and analog process models and of the competing memory models can be found in the excellent review by Widaman et al. (1989).

\section{PRODUCTION VERSUS VERIFICATION TASKS}

In studies investigating the validity of cognitive addition models, the verification-task paradigm usually has been used. The production-task paradigm, in which subjects simply give the answer to the addition problem (e.g., $5+3=$ ?), is rarely used. Nevertheless, most theorists have presented their models as general models for mental addition performance (e.g., Ashcraft, Fierman, \& Bartolotta, 1984; Geary \& Widaman, 1987). They have implicitly assumed that conclusions about the nature of the proposed component processes for verification-task performance are also valid for production-task performance. Ashcraft (Ashcraft, 1983, 1985; Ashcraft et al., 1984) was more explicit when he stated that the longer latencies for "true" verification-task performance (in comparison with latencies for production-task performance) are only due to the extra time needed to make a true/false decision. It is assumed that, theoretically, the only difference between "true" verification-task performance and production-task performance is the absence of the true/false decision process in the production task. Conclusions in relation to the nature of the other processes (and especially in relation to the important search/compute process) are hypothesized to be invariant over different research paradigms. This assumption is supported by Miller, Perlmutter, \& Keating's (1984) study, in which evidence was found for a memory-retrieval process in simple non-tie (i.e., with different addends) addition-production problems.

According to Baroody $(1983,1984,1985)$, however, verification tasks may introduce artifacts into chronometric measures of addition performance. As evidence, he therefore points to some differences in results between studies using a verification and production paradigm. For example, using a production task, Miller et al. (1984) found that RTs of ties were a function of problem size. In verification tasks, however, a problem-size effect of ties has not been found (e.g., Ashcraft \& Stazyk, 1981; Groen \& Parkman, 1972). Furthermore, Ashcraft et al. (1984) found differences between verification and production tasks for adults and fifth graders. While using all 100 basic combinations (ties included), the sum squared model best predicted the verification results, and the minimum addend model best predicted the production results. According to Baroody $(1983,1984,1985)$, these results weaken the retrieval process hypothesis and highlight the importance of counting or other procedural processes in simple mental arithmetic.

A more detailed critique on the use of verification tasks for the construction of a general mental addition model comes from Campbell (1987b). In two experiments using 
single-digit multiplication problems, Campbell (1987b) demonstrated the invalidity of the assumption that presenting the answer has no direct effect on the speed and accuracy of retrieving an answer to the problem. This assumption is essential for generalizing the network-retrievel model to production-task performance. Campbell argued that the presented answer in verification tasks functions as a priming stimulus and that, on "true" verification trials, the effects of priming are sufficient to distort estimates of problem difficulty and to mask important evidence about the nature of the retrieval process. These results are obtained with simple multiplication problems, but there is indirect evidence (LeFevre et al., 1988) that priming also has an effect on addition problems.

\section{THE DECISION-PRODUCTION TASK}

In the present study, we used a new paradigm, a decision-production task, that has the methodological advantages of the verification paradigm (see Campbell, $1987 \mathrm{~b}$ ) and the theoretical advantages of the production paradigm. In the decision-production task, the problems are presented as they are in a production task (e.g., $5+7=$, or $257+761=$ ). The subjects have to generate the answer mentally. But, instead of verbally producing the answer, as is done in an ordinary production task, the subjects must decide whether a particular digit in the generated answer is odd or even. The subject communicates this decision by pressing one of two buttons and afterwards typing the whole answer on a keyboard. The latencies between stimulus onset and odd/even button contact are registered and serve as estimates for the RTs. This procedure is derived from a method used by Pieters (1985), for which subjects had to multiply the digits in the answer of an addition problem and report whether the extreme left digit in the result was odd or even.

A disadvantage of the odd/even decision-production task is that the odd/even decision can be made by means of an odd/even rule (see Krueger \& Hallford, 1984) before actually generating the answer. In such circumstances, no arithmetic processes would be reflected by the RTs. The odd/even rule states (Krueger \& Hallford, 1984) that "if one and only one of the two addends is odd, then the correct sum must be odd; else it must be even"' (p. 171). To prevent subjects from using this strategy, they were instructed explicitly not to check for oddness/evenness before the sum was calculated and were told that the latency between odd/even button contact and typing the solution was registered and had to be small, which is impossible when using an odd/even strategy. The effectiveness of this instruction is supported by the finding that the results of the analyses based only on observations with small latencies between odd/even button and keyboard contact were essentially the same as those on the complete set of observations.

The new demands of the decision-production task required a modification of Widaman et al.'s arithmetic model. In the decision-production task, self-terminating processing is not possible, and a true/false decision stage no longer is needed. Therefore, these assumptions were excluded from the model. On the other hand, the necessity of making an odd/even decision suggests an odd/even decision stage. Other aspects of the Widaman et al. model remained unchanged. Even the assumption of columnwise processing from the right to the left for complex problems was held, because most people spontaneously adopt such a strategy, even if the problems are presented auditorily (Hitch, 1978b).

\section{THE PRESENT STUDY}

We designed two experiments using the decisionproduction paradigm. In both experiments, the problems were presented in a more natural, or mental, arithmeticlike format. That is, they were presented horizontally. In Experiment 1, the model was tested for simple addition problems; in Experiment 2, more complex addition problems were used.

The results will allow us to assess the generalizability of the cognitive components model proposed by Widaman et al. (1989) for simple addition in verification tasks to more complex addition problems and to production tasks. In addition, further information on the relative plausibility of competing conceptualizations of the search/ compute stage will be gained. More specifically, we will determine whether this stage can be characterized in the decision-production task as a memory-retrieval process (as found in most verification studies), rather than as a counting or procedural process (see Baroody, 1985). Furthermore, it will be possible to compare the odd/even decision-production paradigm with the conventional verbal production paradigm.

\section{Experiment 1: Simple Addition}

In Experiment 1, subjects were confronted with the 100 well-known simple addition problems. Because the answers to the simple addition problems used in this experiment consisted of maximally two digits, and the left digit had a very small range $(0-1)$, the odd/even decision had to be made in relation to the right (or unit) digit.

The validity of the Widaman et al. (1989) model for simple addition would be established with the demonstration that regression equations specified to reflect the independent component processes in the task-modified model accurately predict RT. For the generalizability of the retrieval process to addition-production performance, it is necessary to replicate the finding of Miller et al. (1984) and Widaman et al. (1989) that the search/compute process is represented best by the product or sum squared structural variable.

The equivalence of the decision-production task and the conventional production task would require that our results be similar to those of Miller et al. (1984), who analyzed the RTs of the 100 basic addition facts for the conven- 
tional production task. The validity of the paradigm would also require that the odd/even decision does not interact with other processes.

\section{Method}

Subjects. The subjects were 16 graduates in psychology $(10$ males and 6 females). Two subjects were left-handed; the other 14 were right-handed. All subjects participated voluntarily in the experiment.

Material. The set of stimuli consisted of 100 addition problems, all 100 possible pairwise combinations of the integers 0 through 9 (the " 100 basic addition facts"). Thus, ties (i.e., problems with two identical addends; e.g., $3+3$, or $6+6$ ) and non-ties (i.e., problems with two different addends) were included in the set. The problems were presented in a horizontal format (i.e., $p+q=$ ).

Apparatus. A Turbo Pascal 4.0 computer program, running on an IBM PS/2 computer, controlled presentation of problems via an on-line video monitor. The problems appeared centered on the monitor's screen, filling a dotted rectangle $1.50 \mathrm{~cm}$ in height and $5.40 \mathrm{~cm}$ in width. The subject viewed the screen from an eye distance of approximately $70 \mathrm{~cm}$. An IBM keyboard was placed immediately in front of the screen, with the numerical keyboard centered under the vertical middle axis of the screen. All keys except those from the numerical keyboard were covered with a board. Two telegraph keys were placed between the subject and the numerical keyboard, one at each side and at the same distance of the numerical keyboard. The subject responded by pressing one of the telegraph keys and by typing the sum on the keyboard. Corrections were not possible. One of the telegraph keys was labeled even, the other was labeled odd. The positions of the odd and even buttons were counterbalanced across subjects. Latencies were measured and recorded by the computer with $1-\mathrm{msec}$ accuracy from the time the problem was presented on the screen until contact was made on one of the telegraph keys. For measuring RTs, Turbo Pascal timing routines (Brysbaert, Bovens, d'Ydewalle, \& Van Calster, 1989) were used. Latencies between button contact and typing the first digit of the sum were also recorded. Because time measures recorded with a keyboard are slow and unstable (Brysbaert et al., 1989; Graves \& Bradley, 1987), those latencies are not very accurate and do not reflect real-time latencies. The computer also registered incorrect responses.

Design. The experiment consisted of two sessions, separated by a short rest period. In both sessions, the same 100 basic addition problems were presented. The problems were presented randomly, and problems to which the response was incorrect (i.e., a wrong odd/even decision or a wrong sum) were presented again at a random place later in the session. Each problem could appear maximally three times. Only latencies from correct responses were recorded. After each block of 40 trials, the subjects had the opportunity to take a pause.

Procedure. The subjects were tested individually, with each session lasting approximately $10 \mathrm{~min}$. The subjects were asked (1) to compute the sum of the addends, (2) to decide whether the sum was odd or even, (3) to press the appropriate button, (4) to type the sum at the numerical keyboard, and (5) to press the "enter" key. Furthermore, it was stressed that they had to work "as quickly and accurately as possible" and that they first had to make the sum and then had to decide whether the answer was odd or even.

At the beginning of Experiment 1, a practice set of 10 problems was presented. The intersession rest period lasted about $3 \mathrm{~min}$; the interblock rest period lasted $10 \mathrm{sec}$. During this period, the word PAUSE appeared on the screen. The problem-presentation sequence was as follows: (1) a totally black screen appeared for $1 \mathrm{sec}$; (2) together with a short warning signal (a $800-\mathrm{Hz}$ tone during $30 \mathrm{msec}$ ), the white surroundings of a rectangle appeared in the middle of the screen and remained on for $1.5 \mathrm{sec}$; (3) the problem appeared in the rectangle, and the subject pressed one of the buttons and typed the sum on the keyboard; (4) the problem remained on the screen, and the digits of the sum appeared one by one in the rectangle on the screen; and (5) when the sum was complete, the subject pressed the "enter" key, and the next trial was presented. However, if the subject responded incorrectly, there was an extra step: Immediately after the subject typed "enter," a black screen with the word WRONG appeared for $1 \mathrm{sec}$

\section{Results and Discussion}

At the first presentation, the overall error rate in the matrix of 3,200 trials was $5.44 \%(5.63 \%$ and $5.25 \%$ for the first and the second session, respectively). The RTs of these error trials were not recorded, but instead of excluding them from further analyses, the procedure of presentation provided a second and eventually a third possibility to get correct RTs to these problems. As a result, the rate of missing values in the matrix of 3,200 trials due to errors was $0.09 \%$. This technique of data sampling can be justified by the fact that most errors at first presentation ( $81 \%$ of all errors) were the result of a wrong odd/even decision when the sum was correct. Analyses were carried out on RT data of Sessions 1 and 2 and on the combined data of the two sessions. Because there were no significant differences between the results from Sessions 1 and 2, only the analyses of the combined data will be reported.

Information-processing models of mental addition were formulated in mathematical terms by means of regression equations specifying structural variables for the proposed component processes in the model. The models were fit to the RT data using hierarchical regression techniques (Cohen \& Cohen, 1975). Structural variables for the search/compute process included the product of the addends (Prod) (Geary \& Widaman, 1987; Miller et al., 1984; Widaman et al., 1989), the square of the sum $\left(\mathrm{Sum}^{2}\right)$ (Ashcraft, 1982), and the five counting-based variables proposed by Groen and Parkman (1972): the minimum addend (Min), the maximum addend (Max), the sum of addends (Sum), the first addend (Addendi), and the second addend (Addend2). The structural variable for the odd/even decision process was the odd/even (OE) variable, which represented possible intercept differences between odd and even problems. In the absence of any cognitive models for odd/even judgments, the $O E$ variable was arbitrarily coded: 0.5 for odd, and -0.5 for even problems. Structural variables for the encoding and the response processes could not be constructed. As a result, speed of encoding digits and time to prepare a response were incorporated into the intercept term.

Correlations between the different structural variables and mean RTs are presented in Table 1. Correlations were computed separately for tie and non-tie problems. The analyses of tie problems (see the above-diagonal entries in Table 1) showed a ties effect. That is, there was no relation between problem size and RT for ties. This result is consistent with the results in verification tasks (Ashcraft \& Battaglia, 1978; Groen \& Parkman, 1972) but is 
Table 1

Experiment 1: Correlation Matrix for Reaction Time Variables and Structural Variables

\begin{tabular}{|c|c|c|c|c|c|c|c|c|c|c|c|}
\hline Variable & 1 & 2 & 3 & 4 & 5 & 6 & 7 & 8 & 9 & 10 & 11 \\
\hline \multicolumn{12}{|c|}{ Reaction Time Variables } \\
\hline $\begin{array}{l}1 \\
2 \\
3\end{array}$ & $\begin{array}{l}.863 \\
.966\end{array}$ & .964 & $\begin{array}{l}.984 \\
.967\end{array}$ & $\begin{array}{r}-.150 \\
.364 \\
-.244\end{array}$ & $\begin{array}{r}-.150 \\
.364 \\
-.244\end{array}$ & $\begin{array}{r}-.150 \\
.364 \\
-.244\end{array}$ & $\begin{array}{r}.061 \\
.183 \\
-.040\end{array}$ & $\begin{array}{r}.061 \\
.183 \\
-.040\end{array}$ & $\begin{array}{r}-.150 \\
.364 \\
-.244\end{array}$ & $\begin{array}{r}-.150 \\
.364 \\
-.244\end{array}$ & $\begin{array}{l}- \\
- \\
-\end{array}$ \\
\hline \multicolumn{12}{|c|}{ Structural Variables } \\
\hline 4 & .661 & .672 & .691 & & 1.000 & 1.000 & .963 & .963 & 1.000 & 1.000 & - \\
\hline 5 & .437 & .537 & .504 & .500 & & 1.000 & .963 & .963 & 1.000 & 1.000 & - \\
\hline 6 & .634 & .698 & .690 & .866 & .866 & & .963 & .963 & 1.000 & 1.000 & - \\
\hline 7 & .689 & .745 & .743 & .900 & .781 & .971 & & 1.000 & .963 & .963 & - \\
\hline 8 & .703 & .733 & .744 & .970 & .607 & .910 & .966 & & .963 & .963 & - \\
\hline 9 & .464 & .438 & .467 & .577 & .577 & .667 & .647 & .607 & & 1.000 & - \\
\hline 10 & .382 & .493 & .453 & .577 & .577 & .667 & .647 & .607 & -.111 & & - \\
\hline 11 & .153 & .146 & .155 & .067 & -.067 & .000 & .021 & .039 & .000 & .000 & - \\
\hline
\end{tabular}

Note-1 = RT for Session 1; $2=$ RT for Session 2; $3=$ Mean RT for Sessions 1 and $2 ; 4=$ smaller stimulus addend; $5=$ larger stimulus addend; $6=$ sum of stimuli addends; $7=$ square of sum of stimuli addends; 8 product of stimuli; $9=$ first stimulus addend; $10=$ second stimulus addend; $11=$ odd/even for sum (coded -0.5 for even, 0.5 for odd). Above-diagonal entries are based on the 10 ties; belowdiagonal entries represent the 90 pairs of non-tie stimuli. For the below-diagonal entries, critical values are $[r(88)=.207, p<.05 ; r(88)=.270, p<.01]$. For the above-diagonal entries, critical values are $[r(8)=.632, p<.05 ; r(8)=.765, p<.01]$.

contradictory to the finding of Miller et al. (1984), who observed a positive relation between RT and size of addends of addition ties in a production task. Further inspection of the ties RTs, however, reveals that the ties effect is mainly due to the very high RT to the $0+0$ tie. The mean RT to the $0+0$ tie was $956 \mathrm{msec}$, whereas the mean RT of the other ties was only $732 \mathrm{msec}$. When this $\mathrm{RT}$, which is probably inflated through the unnatural task of evaluating the evenness of the 0 digit, was deleted from the ties data set, we found an effect of problem size: The correlation between the Prod variable and the RT was moderately high $[r(7)=.41]$, but not significant. The similarity between the RT pattern of the ties in our decision-production task and in Miller et al.'s (1984) study $[r(8)=.59]$, however, provides a first indication of the equivalence of the decision-production task to a normal production task.

For the 90 non-ties (see the below-diagonal entries in Table 1), RT was positively correlated with problem size: All correlations between $\mathrm{RT}$ and the problem-size variables were significant $(p<.01)$. The use of $t$ tests for dependent correlations (Cohen \& Cohen, 1975) revealed that the correlations between RT and the Addend1, Addend2, and Max structural variables were all significantly different $(p<.05)$ from the correlations between RT and the other problem-size structural variables. Separate analyses for Sessions 1 and 2 gave identical results. The Max, Addend1, and Addend 2 variables seemed to have been less important variables in predicting RTs in mental addition tasks (see also, e.g., Ashcraft \& Battaglia, 1978; Groen \& Parkman, 1972), and were therefore excluded from further analyses.

The validity of the componential model. Hierarchical regression techniques were used to identify an optimal model for simple mental addition and to evaluate the a pri- ori proposed mental addition model. Regression models for simple addition were fit using each of the four important search/compute variables and the $\mathrm{OE}$ variable. Furthermore, the model fits for elementary simple addition regression models $(R T=a+b$ [search/compute variable]) were compared with fits of extended simple addition models that included the odd/even decision parameter ( $R T=a+b_{1}$ [search/compute variable] $+b_{2}$ [odd/even variable]). The increase in the $R^{2}$ associated with the addition of the $O E$ variable was tested using an incremental $F$ test (Cohen \& Cohen, 1975).

These analyses revealed that adding the $\mathrm{OE}$ variable significantly increased the regression model $R^{2}$ in the Sum ${ }^{2}$ and Sum regression equations [increment ratios were, respectively, $F(1,87)=3.952, p<.05$, and $F(1,87)=$ 4.190, $p<.05$ ]. The increments in the Prod and Min model were not significant [respectively, $F(1,87)=$ $3.201, p>.05$, and $F(1,87)=2.014, p>.15]$. When analyzing the data for Sessions 1 and 2 separately, the increments never were significant $(p>.05)$. Thus, the intercept difference between an "odd" and an "even" judgment is too small to reach significance reliably (the mean RTs of the odd and even problems were, respectively, 859 and $816 \mathrm{msec}$, and the $\mathrm{OE}$ parameter estimates in the different models varied from 30 to $39 \mathrm{msec}$ ), indicating that the statistical and psychological importance of the $\mathrm{OE}$ variable is minimal. Therefore, the $\mathrm{OE}$ parameter was excluded from the final regression models for simple addition problems, and the difference in time to make an odd or even decision was hypothesized to be incorporated into the intercept value.

Summary results for the three best full model regression equations are presented in Table 2 . The reasonably good fits for these componential models support the hypothesis that the Widaman et al. model is also valid for 
Table 2

Experiment 1: Statistical Summaries of Regression Analyses

\begin{tabular}{|c|c|c|c|c|c|}
\hline Equation & Partial $F$ & $R^{2}$ & $F$ & $d f$ & Root $M S_{\mathrm{e}}$ \\
\hline $\mathrm{RT}=736+5.39$ (Prod) & 109.18 & .554 & 109.17 & 1,88 & 92.73 \\
\hline $\mathrm{RT}=703+1.44\left(\mathrm{Sum}^{2}\right)$ & 108.53 & .552 & 108.54 & 1,88 & 92.88 \\
\hline $\mathrm{RT}=726+42.90(\mathrm{Min})$ & 80.46 & .478 & 80.46 & 1,88 & 100.32 \\
\hline
\end{tabular}

Note - Prod $=$ product $; \mathrm{Sum}^{2}=$ square of the correct sum; Min $=$ smaller of two addends. All models and partial $F$ ratios were significant at the $p<.01$ level.

simple production-task performance. The superiority of the Prod and Sum ${ }^{2}$ structural variables in predicting RTs in simple mental addition tasks, for both the decisionproduction paradigm (the present experiment) and the verification paradigm (Geary et al., 1986; Miller et al., 1984), demonstrates the similarity between the performance in production and verification tasks and provides evidence for the generalizability of the network-retrieval hypothesis. This conclusion is counter to Baroody's (1983, 1984,1985 ) opinions that the evidence for the retrieval model is task-specific and that mental addition involves reconstructive processes and procedural knowledge. It also suggests that intraitem priming effects in additionverification tasks do not distort the retrieval process in an important way, at least not in non-tie problems.

The equivalence of the decision-production paradigm and the conventional production paradigm. The comparability of the present results with the results of the Miller et al. (1984) study suggests that the decisionproduction task is a valuable alternative to the verbal production task: To further validate the decision-production paradigm, we investigated whether an odd or even decision had a differential effect on the retrieval process. That is, we tested the Prod model-which was chosen as the final cognitive model for simple mental addition-for odd and even problems separately and looked for a possible interaction effect. The results revealed a marginally significant Prod $\times$ OE interaction effect $[F(1,86)=4.277$, $p<.05]$. Separate analyses of the data from Sessions 1 and 2 revealed a significant interaction effect for Session $2[F(1,86)=6.376, p<.05]$. The interaction effect in Session 1, however, was not significant $[F(1,86)=$ $1.812, p>.15]$. Altogether, these results do not exclude the possibility that an odd or even decision may have a differential effect on the retrieval process and on simple addition RT. Therefore, further investigations will be necessary in order to completely evaluate the odd/even decision-production paradigm.

\section{Experiment 2: Complex Addition}

In Experiment 2, we checked whether the task-modified model of Widaman et al. (1989) is also valid for addition problems that are more complex (three-digit integers) than the most complex problems (two-digit integers) used by Widaman (Geary \& Widaman, 1987; Widaman et al., 1989). In the decision-production task used in the present experiment, subjects had to decide whether the second digit from the right (i.e., the tens digit) was odd or even.
This requirement made it difficult for the subjects to use an odd/even strategy.

\section{Method}

Subjects. The subjects were the same 16 graduates in psychology as in Experiment 1. Experiment 2 took place 2 days before Experiment 1

Material. A set of 180 complex addition problems served as stimuli. Each of the 180 complex addition problems consisted of two three-digit integers presented in a horizontal format (i.e., abc + def $=$ ). The problems were generated randomly with the following constraints: (1) all three-digit integers had to be integers between 100 and 999; (2) within a given problem, all six digits had to be unique; (3) for one third of the problems, the sum of the units column and the sum of the tens column was not allowed to be greater than 9 (i.e., no carry operation was required); for another third of the problems, the sum of the units column or the sum of tens column had to be greater than 9 (one carry operation was required); for the last third of the problems, both sums had to be greater than 9 (two carry operations were required to solve the problem); (4) problems with a units sum greater than 9 and a tens sum of 9 were not allowed (these are problems that require two carry operations that are not independent of each other); (5) for the problems with one carry operation, the carry operation had to appear in the units column for one half the problems and in the tens column for the other half; (6) in each of the above "carry" conditions, for one half of the problems, the penultimate digit of the correct sum had to be even; for the other half, this digit had to be odd; and (7) the larger integer had to be the first addend for one half of the problems in each condition.

Apparatus and Procedure. The apparatus and procedure were the same as in Experiment 1, except that the subjects were required to decide whether the tens digit of the sum was odd or even. Experiment 2 lasted about $1 \mathrm{~h}$.

Design. All 180 problems were presented in one session in a random order. After each block of 40 trials, the subjects had the opportunity to take a pause. During the first three pauses, the subjects were confronted with a problem written on a card and were asked to compute the sum and to verbalize the steps they used. As in Experiment 1, problems responded to incorrectly were presented again at a random place later in the session.

\section{Results and Discussion}

At the first presentation, overall error rate in the matrix of 2,880 trials was high $(12.36 \%)$. The presentation procedure, however, provided an RT data matrix that was almost complete: The rate of missing values due to errors was $0.45 \%$ (the number of missing values per subject varied from 0 through 3). Statistical analyses were performed on mean RTs, with each mean RT minimally based on 15 trials.

Mathematical models for complex addition problems were formulated according to the assumptions and process 
components specified by the modified Widaman et al. model. Again, these process models were fit to RT data using hierarchical regression techniques. Structural variables for the search/compute process were the seven search/compute variables used in Experiment 1: Addend1, Addend2, Max, Min, Sum, Sum ${ }^{2}$, and Prod. In Experiment 2, however, the search/compute variables were defined for each column separately. For example, the Prod variable representing a search retrieval process actually consisted of three column structural variables: the units column product (Uprod), the tens column product (Tprod), and the hundreds column product (Hprod). The hypothesized carry component process was also simultaneously represented by two structural variables: the unit carry variable (Ucarry) and the tens carry variable (Tcarry). The Ucarry and Tcarry variables were coded 0 for the absence and 1 for the presence of a carry from, respectively, the units column to the tens column, and the tens column to the hundreds column. The $\mathrm{OE}$ variable representing the intercept difference between odd and even decisions was coded 0.5 for odd and -0.5 for even problems. The other processes (e.g., response, encoding, decision) could not be represented by a structural variable and were therefore incorporated into the intercept.

The validity of the componential model. Independent regression models for the complex addition problems for each of the seven search/compute structural variables were fit using the units, tens, and hundreds search/compute variables, the Ucarry and Tcarry structural variables, and the $\mathrm{OE}$ variable. As in Experiment 1, the Min, $\mathrm{Sum}^{2}$, and Prod models gave the best fits (respectively, .738, .746, and .742). Therefore, only analyses in relation to these three search/compute variables are reported. Hierarchical regression analyses revealed the nonsignificance of the Ucarry parameter $(p>.05)$ and the OE parameter $(p>.05)$ in all three models. Summary results for the three best full model regression equations are presented in Table 3.

In the equations presented in Table 3, the search/compute structural variables were estimated separately for each column. Contradictory to the results of Widaman et al. (1989), we found that the columnwise search/compute slope estimates were not identical. Multiple regression analyses, with the restriction that the slope estimates for the columnwise search/compute variables had to be equal, were performed for each model. For all three models, incremental $F$ tests showed that the decrease in $R^{2}$ associated with enforcing the equality constraints was significant [for the Prod model, $F(2,175)=7.185, p<.01$; for the Sum ${ }^{2}$ model, $F(2,175)=7.488, p<.01$; and for the Min model $F(2,175)=5.129, p<.01]$. These results suggest that the search/compute process requires a different amount of time depending on the column that is processed. Inspection of the equations presented in Table 3, however, reveals that the hundreds and tens parameter are highly similar, but that both differ from the units parameter. We tested this hypothesis by constraining the slope estimates for the tens and hundreds columns to be equal. Incremental $F$ tests revealed that the decrease in $R^{2}$ associated with the equality constraint was never significant [for the Prod model, $F(1,175)=.005, p>.90$; for the Sum ${ }^{2}$ model, $F(1,175)=1.020, p>.30$; and for the Min model, $F(1,175)=2.396, p>.10]$. The Prod model was most in accordance with the equality constraints.

Because the three structural variables are highly intercorrelated, the different models could not be differentiated statistically. The slightly higher fits of the $\mathrm{Sum}^{2}$ and Prod models, however, indicate the superiority of a memoryretrieval hypothesis. As in Experiment 1, the search/compute process can be characterized as a memory retrieval or a search process. Taking into account the fact that the

Table 3

Experiment 2: Statistical Summaries of the Three Best Full-Regression Models for the Three Best Search/Compute Column Variables for Complex Addition

\begin{tabular}{|c|c|c|c|c|}
\hline Equation & $R^{2}$ & $F$ & $d f$ & Root $M S_{\mathrm{e}}$ \\
\hline $\begin{array}{c}\text { RT }=3,558+19.0 \text { (Hprod) }+18.6 \text { (Tprod) } \\
+1,780 \text { (Tcarry) }+35.0 \text { (Uprod) } \\
\text { (Partial Fs: } 29.99,9.33,56.42,100.24)\end{array}$ & .7394 & 124.148 & 4,175 & 879.29 \\
\hline $\begin{array}{c}\mathrm{RT}=3,047+5.78\left(\mathrm{Hsum}^{2}\right)+3.91\left(\mathrm{Tsum}^{2}\right) \\
+1,885(\mathrm{Tcarry})+9.66\left(\mathrm{Usum}^{2}\right) \\
\text { (Partial Fs: 39.39, 4.98, 54.32, 104.06) }\end{array}$ & .7394 & 124.111 & 4,175 & 879.38 \\
\hline $\mathrm{RT}=3,355+145(\mathrm{Hmin})+231(\mathrm{Tmin})$ & .7373 & 122.805 & 4,175 & 882.82 \\
\hline
\end{tabular}

$+1,550$ (Tcarry) +276 (Umin)

(Partial Fs: $18.96,24.22,51.67,85.54)$

$\overline{\mathrm{RT}}=6096$

Note-Umin = units minimum; Uprod $=$ units product; Usum $^{2}=$ units sum squared; Tmin $=$ tens minimum; Tprod = tens product; Tsum $^{2}=$ tens sum squared; Hmin = hundreds min; Hprod $=$ hundreds product; Hsum $^{2}=$ hundreds sum squared; Tcarry $=$ tens carry. $\quad$ All models were significant $(p<.0001)$; all partial $F$ ratios were significant at $p<.01$, except the $F$ ratio of the Tsum ${ }^{2}$ parameter, which was significant at $p<.05$. 
Table 4

\begin{tabular}{|c|c|c|c|c|}
\hline Equation & $R^{2}$ & $F$ & $d f$ & Root $M S_{\mathrm{c}}$ \\
\hline \multicolumn{5}{|c|}{ Across All Problems } \\
\hline $\begin{array}{c}\mathrm{RT}=3,560+18.9 \text { (Hprod) }+18.9 \text { (Tprod) } \\
+1,768 \text { (Tcarry) }+35.0 \text { (Uprod) } \\
\text { (Partial Fs: } 36.14,36.14,120.89,101.89)\end{array}$ & .7394 & 166.471 & 3,176 & 875.80 \\
\hline \multicolumn{5}{|c|}{ "Odd" Problems } \\
\hline $\begin{array}{c}\mathrm{RT}=3,536+22.4 \text { (Hprod) }+22.4 \text { (Tprod) } \\
+1,459 \text { (Tcarry) }+33.5 \text { (Uprod) } \\
\text { (Partial Fs: } 21.46,21.46,31.98,40.03)\end{array}$ & .7125 & 71.058 & 3,86 & 910.83 \\
\hline \multicolumn{5}{|c|}{ “Even” Problems } \\
\hline $\begin{array}{c}\mathrm{RT}=3,560+16.5 \text { (Hprod) }+16.5 \text { (Tprod) } \\
+2,017 \text { (Tcarry) }+36.5 \text { (Uprod) } \\
\text { (Partial Fs: } 15.53,15.53,97.73,65.55)\end{array}$ & .7750 & 98.746 & 3,86 & 839.84 \\
\hline
\end{tabular}

Prod model has been consistently one of the best predictive models in different studies (Geary et al., 1986; Miller et al., 1984), the product model with equal hundreds and tens search/compute parameter estimates was chosen as the final model. A statistical summary of this model is presented in Table 4 . To test the independence of the carry process from the search/compute process, product $x$ carry interactions were estimated for each column, and the significance of these interactions was tested with an incremental $F$ test. The addition of these interactions to the equation resulted in a nonsignificant increase in the full model $R^{2}[F(3,173)=1.826, p>.10]$. The independence of the Tcarry process was also supported by the finding that the regression estimate for the Tcarry variable was almost identical across the three equations that include the Prod, $\mathrm{Sum}^{2}$, and Min variables, respectively (see Table 3).

Altogether, these results give evidence for the basic aspects of the Widaman et al. componential model: the presence of search/compute processes and a carry process, and the independence of both of these processes. Some violations to the model will be discussed in a later section.

The effect of the odd/even decision in a production paradigm. We evaluated the validity of the OE decisionproduction paradigm by testing the final product model separately for odd and even problems. Summary results are presented in Table 4 . In both conditions, model fits were high and of a comparable magnitude, the decrease in fits by the hundreds/tens parameter equality constraints were not significant $(p>.50)$, and a test for homogeneity of parameter estimates of the two regression equations (Rao, 1973) revealed no significant difference $[F(4,170)=0.930, p>.30]$. We also extended the final product model with the $\mathrm{OE}$ variable and all columnwise $\mathrm{OE} \times$ Prod interactions and the $\mathrm{OE} \times$ Tcarry interaction. An incremental $F$ test revealed that the inclusion of these variables was nonsignificant $[F(5,171)=0.861$, $p>.30]$. Moreover, not one of these additional param- eters was significant $(p>.15)$. These results indicate that the OE judgment, typical for the decision-production task, has no differential effect on the RT and does not influence other processes in the model. The equivalence of the decision-production paradigm to the conventional production paradigm is thereby supported.

The role of short-term memory load and strategy choice. As the results of the regression analyses indicate, the proposed model for complex addition was not totally validated. The most deviant finding in our analyses of complex addition problems is the absence of the Ucarry process. It is possible that the hypothesized Ucarry process requires no time or does not have to be executed at all to solve the complex problems. This hypothesis is supported by the introspective reports of some of the subjects. During Experiment 2, introspective reports on the solution of a problem were collected three times. These reports reveal that 5 out of 16 subjects sometimes processed the units and tens columns simultaneously (e.g., when confronted with the problem $678+365=$, they transformed the subaddition $78+65$ to $138+5$ ). This strategy resembles a chunking strategy. Carroll (1976) described this strategy as "mentally adding two-digit numbers both at a time rather than by the more 'elementary' one-digit-and-carry methods"' (p. 50). The nonsignificance of the Ucarry parameter can be effected by this strategy of simultaneous processing. However, separate analyses for both strategy groups gave no differences in relation to the Ucarry parameter: In both groups, the Ucarry parameter was not significant. Therefore, the nonsignificance of the Ucarry can not be the result of the chunking strategy alone.

The introspective reports, however, give support for another explanation for the nonsignificance of the Ucarry parameter. The verbal reports of the subjects revealed that 11 out of the 16 subjects had usually used a hundredstens-units (HTU) processing strategy. If we conceptualize the carry parameter as the time necessary to "mentally set aside the value one" or to "hold the value one," 
the HTU strategy makes it possible to omit the Ucarry process. In a HTU strategy, where the units column is processed last, the total sum can be constructed immediately after retrieving the units column sum. For example, after processing the hundreds and tens columns, the problem $678+365=$ is transformed into the problem $1030+(8+5)$. After retrieving the unit column sum (i.e., 13), the combination of the units sum and the partial hundreds-tens sum can be made directly without the execution of a Ucarry or hold process. This $H T U$-withoutUcarry strategy, which is consistent with a columnwise processing and a chunking strategy, is probably more efficient than is a strategy with a Ucarry process.

Another surprising finding also becomes more plausible in the light of a HTU processing strategy. On the basis of the results of Widaman et al. (1989) and Geary et al. (1986), we expected to find almost equal parameter estimates for the different column retrieval processes. But, as shown by the independent regression equations in Table 3, the parameter estimates for the different column retrieval processes differ from each other. More specifically, the parameter estimate for the unit retrieval process is much higher than are the parameter estimates for the hundreds and tens retrieval process (see, e.g., the final model, Table 4). This difference between parameter estimates cannot be interpreted when a right-to-left or unitstens-hundreds (UTH) processing strategy is assumed. Under the assumption of an HTU processing strategy, however, the larger Uprod parameter can be the result of the delaying effect of short-term memory, where partial results of the processing of the hundreds and tens columns are stored. An important characteristic of arithmetic production tasks (contrary to verification tasks) is the fact that subjects have to take partial results into account. Jacobs and Lévy-Schoen (1988) demonstrated the delaying effect of short-term memory load in a visual dual task. They found that the duration of a "combination process" increased with task difficulty, which was mainly affected by memory set size.

The importance of short-term memory and the storage of partial results in arithmetic production tasks also have been demonstrated by Hitch (1978a, 1978b) and Dansereau and Gregg (1966). Dansereau and Gregg showed that the hold process is one of the primary processes in complex mental multiplication. Hitch (1978b) found that errors in complex addition problems are due to rapid forgetting of interim information or partial results. Research on cognitive processes in mental arithmetic has usually neglected these processes. The widespread use of verification tasks and the lack of studies on more complex problems are mainly responsible for this neglect.

As mentioned above, the columnwise and chunking strategy groups cannot be differentiated by the Ucarry parameter. As for the other processes, however, there are a lot of differences between the chunking and columnwise groups. The results of the columnwise processing group are similar to the results of the analyses on data of all of the subjects. The final model equation for this group is the following: RT $=3,392+19.01$ (Hprod) +20.11 (Tprod) $+1,356$ (Tcarry) +28.58 (Uprod); $R^{2}=.709 ; F(4,175)=106.811$. The final model for the chunking group however, took the form of: $\mathrm{RT}=4,044$ +17.74 (Hprod) $+3,197$ (Tcarry) +49.17 (Uprod); $R^{2}=.5905 ; F(3,176)=84.587$. The most surprising finding in the chunking group is the absence of a Tprod parameter, which, together with the finding of a much higher Tcarry parameter, means that in a tens-units chunking strategy the addition of the tens column takes a constant amount of time (incorporated into the intercept) plus an extra amount of time needed when the tens sum is greater than 9-this extra time is incorporated into the Tcarry parameter. The absence of an addend-size effect in the tens column is consistent with the different nature of the chunking strategy, for which addition of the tens column means addition of two two-digit addends. Another conspicuous finding is the very high Uprod parameter. Besides the delaying effect of the partial sum, the Uprod parameter can be inflated by the fact that in a chunking strategy the addition of the units column corresponds with the addition of a one-digit addend with a three- or twodigit addend. In the units column, unlike in the tens column, an addend-size effect can be expected because one of the addends is always a one-digit addend.

\section{GENERAL DISCUSSION}

The results of the present study support most of the basic aspects of the Widaman et al. (1989) model. Evidence was found for the most important arithmetic component processes in the Widaman et al. model: a search/compute and a carry process. Moreover, the present study supported the identification of the search/compute process as a process that involves the retrieval of arithmetic facts from a long-term memory network. The evidence for a memory-retrieval process in production tasks and in very complex addition problems gives further support for the validity and generality of this process in a general model of mental addition. Moreover, this evidence argues against hypotheses that claim the importance of counting or reconstructive processes in adult mental addition (Baroody, $1983,1984,1985$ ). The comparability of the present results with results of studies using a verification paradigm (Ashcraft \& Stazyk, 1981; Widaman et al., 1989) also suggests that possible priming effects in verification tasks do not distort the retrieval process in any important way. However, more detailed studies on the role of priming in arithmetic tasks are needed.

The identification of a carry process in the solution of complex addition problems is consistent with other studies on complex addition (Ashcraft \& Stazyk, 1981; Geary et al., 1986; Hamann \& Ashcraft, 1985; Hitch, 1978a, 1978b). It seems that the intuitive idea of the importance of carry stages in the solution of complex addition problems is correct. Furthermore, the present study supported the inherent assumptions of the componential model: the assumption of mutual independence of the 
component processes and the assumption of serial processing. The high fits of the additive processing models are congruent with the widespread opinion that multidigit calculations are carried out in a series of elementary stages. Evidence for this stage hypothesis in very complex addition problems was previously reported by Hitch (1978b) for auditorily presented addition problems.

Although the present study provides evidence for the basic components of the Widaman et al. model, some less essential assumptions and findings of Widaman et al. were not supported. In our task with complex additionproduction problems presented in a horizontal format, the assumption of right-to-left or UTH columnwise processing could not be supported. Introspective reports strongly supported the use of a HTU strategy when solving horizontal format problems. Further investigations into the differences between the two sequence strategies, however, were not possible, because both strategies could not be formulated in a different mathematical manner.

The evidence for the use of still other strategies suggests that the assumption of strict columnwise processing also is not always valid in horizontal format problems. Moreover, our results suggest that strategy choice and memory load can have an influence on information-processing characteristics. Particularly, some results indicate that memory load or the storage of partial results can have a delaying effect on the arithmetic search/compute process and that some strategy choices make some processing stages superfluous. These findings must be investigated further, but it is clear that short-term memory load and strategy choice play an important role in complex additionproduction tasks. Therefore, a "general" model of mental arithmetic must take into account these memory aspects and differences in strategy use and strategy choice. Recent models in the domain of children's simple arithmetic (Ashcraft, 1987; Siegler, 1988) are good examples of models that have incorporated these aspects in a general model.

Finally, the results obtained with the odd/even decisionproduction tasks were highly similar to the results of studies using a verbal production task (Miller et al., 1984). For non-tie simple addition problems, our study, as well as Miller's study, gave evidence for a model in which the search/compute stage was characterized as a memory-retrieval process. For tie problems, our results were more consistent with production-task performance than with verification-task performance. Besides the comparability between the decision-production and verbal production tasks, the validity of the decision-production task was also demonstrated by the finding that an "odd" or "even" decision had no differential effect on the cognitive processes of the proposed model.

To conclude, the present study provides evidence for the generalizability of the basic assumptions of the Widaman et al. (1989) model to production-task performance and to more complex addition problems. The study also provides further evidence for a memory-retrieval process for simple addition facts. Furthermore, it suggests that performance in more naturalistic addition tasks is influenced by memory load factors and strategy choices and that people differ in relation to the strategies they use. Finally, the validity of the decision-production research paradigm in the study of mental arithmetic was demonstrated.

\section{REFERENCES}

Anderson, J. R. (1983). The architecture of cognition. Cambridge, MA: Harvard University Press.

AshCraft, M. H. (1982). The development of mental arithmetic: A chronometric approach. Developmental Review, 2, 213-236.

Ashcraft, M. H. (1983). Procedural knowledge versus fact retrieval in mental arithmetic: A reply to Baroody. Developmental Review, 3 , 231-235

AsHCRAFT, M. H. (1985). Is it farfetched that some of us remember our arithmetic facts? Joumal for Research in Mathematics Education, 16, 99-105.

AsHCRAFT, M. H. (1987). Children's knowledge of simple arithmetic: A developmental model and simulation. In J. Bisanz, C. J. Brainerd, \& R. Kail (Eds.), Formal models in developmental psychology (pp. 302-338). New York: Springer-Verlag.

AshCraft, M. H., Battaglia, J. (1978). Cognitive arithmetic: Evidence for retrieval and decision processes in mental addition. Journal of Experimental Psychology: Human Learning \& Memory, 4, 527-538

Ashcraft, M. H., Fierman, B. A., \& Bartolotta, R. (1984). The production and verification tasks in mental addition: An empirical comparison. Developmental Review, 4, 157-170.

AshCraft, M. H., \& STAZYK, E. H. (1981). Mental addition: A test of three verification models. Memory \& Cognition, 9, 185-196.

BAroOdY, A. J. (1983). The development of procedural knowledge: An alternative explanation for chronometric trends of mental arithmetic. Developmental Review, 3, 225-230.

BAROODY, A. J. (1984). A re-examination of mental arithmetic models and data: A reply to Ashcraft. Developmental Review, 4, 148-156.

BAROODY, A. J. (1985). Mastery of basic number combinations: Internalization of relationships or facts? Journal for Research in Mathematics Education, 16, 83-98.

Brysbaert, M., Bovens, N., d'Ydewalle, G., Van Calster, J. (1989). Turbo pascal timing routines for the IBM microcomputer family. Behavior Research Methods, Instruments, \& Computers, 21, 73-83.

CAMPBeLL, J. I. D. (1987a). Network interference and mental multiplication. Journal of Experimental Psychology: Learning, Memory, \& Cognition, 13, 109-123.

CAmpBell, J. I. D. (1987b). Production, verification, and priming of multiplication facts. Memory \& Cognition, 15, 349-364.

Carrol, J. B. (1976). Psychometric tests as cognitive tasks: A new "structure of intellect." In L. B. Resnick (Ed.), The nature of intelligence (pp. 27-56). New York: Wiley

COHEN, J., \&OHEN, P. (1975). Applied multiple regression/correlation analysis for the behavioral sciences (lst ed.). Hillsdale, NJ: Erlbaum

Coluss, A. M., LofTus, E. F. (1975). A spreading-activation theory of semantic processing. Psychological Review, 82, 407-428.

Dansereau, D. F., \& GreGG, L. W. (1966). An information processing analysis of mental multiplication. Psychonomic Science, 6, 71-72.

GeARY, D. C., \& Widaman, K. F. (1987). Individual differences in cognitive arithmetic. Joumal of Experimental Psychology: General, 116, 154-171.

Geary, D. C., Widaman, K. F., \& Little, T. D. (1986). Cognitive addition and multiplication: Evidence for a single memory network. Memory \& Cognition, 14, 478-487.

Graves, R., Bradley, R. (1987). Millisecond interval timer and au- 
ditory reaction time programs for the IBM PC. Behavior Research Methods, Instruments, \& Computers, 19, 30-35.

Groen, G. J., Parkman, J. M. (1972). A chronometric analysis of simple addition. Psychological Review, 79, 329-343

HamanN, M. S., \& AshCraft, M. H. (1985). Simple and complex mental addition across development. Journal of Experimental Child Psychology, 40, 49-72.

Hıтсн, G. J. (1978a). Mental arithmetic: Short-term storage and information processing in a cognitive skill. In A. M. Lesgold, J. W. Pellegrino, S. D. Fokkema, \& R. Glaser (Eds.), Cognitive psychology and instruction (pp. 331-338). New York: Plenum.

HrTcH, G. J. (1978b). The role of short-term working memory in mental arithmetic. Cognitive Psychology, 10, 302-323.

JACOBS, A. M., \& LÉvY-SCHOEN, A. (1988). Breaking down saccade latency into central and peripheral processing times in a visual dualtask. In G. Lüer, U. Lass, \& J. Shallo-Hoffman (Eds.), Eye movement research: Physiological and psychological aspects (pp. 267-285). Toronto: Hogrefe.

Krueger, L. E., Hallford, E. W. (1984). Why $2+2=5$ looks so wrong: On the odd-even rule in sum verification. Memory \& Cognition, 12, 171-180.

LeFevre, J.-A., Bisanz, J., Mrxonjic, L. (1988). Cognitive arithmetic: Evidence for obligatory activation of arithmetic facts. Memory \& Cognition, 16, 45-53.
Miller, K., Perlmutter, M., Keating, D. (1984). Cognitive arithmetic: Comparisons of operations. Journal of Experimental Psychology: Learning, Memory, \& Cognition, 10, 46-60.

Pieters, J. P. M. (1985). Reaction time analysis of simple mental tasks: A general approach. Acta Psychologica, 59, 227-269.

RAO, C. R. (1973). Linear statistical inference and its applications (2nd ed.). New York: Wiley.

SIEGLER, R. S. (1988). Strategy choice procedures and the development of multiplication skill. Joumal of Experimental Psychology: General, 117, 258-275.

Stazyk, E. H., Ashcraft, M. H., \& Hamann, M. S. (1982). A network approach to mental multiplication. Joumal of Experimental Psy. chology: Learning, Memory, \& Cognition, 8, 320-335.

SVENSON, O. (1985). Memory retrieval of answers of simple additions as reflected in response latencies. Acta Psychologica, 59, 285-304.

Widaman, K. F., Geary, D. C., Cormier, P., \& LitTle. T. D. (1989). A componential model for mental addition. Journal of Experimental Psychology: Learning, Memory, \& Cognition, 15, 898-919.

(Manuscript received December 27, 1988; revision accepted for publication September 19, 1989.) 